\title{
Reduction in Proportion of Patients With Severe Pain Following Herniorrhaphy Using HTX-OI I as the Foundation of a Non-Opioid Multimodal Analgesic Regimen
}

Neil Singla, MD,' Barry Quart, PharmD, ${ }^{2}$ Jackie Evans-Shields, PharmD, ${ }^{2}$ Jia Hu, PhD, ${ }^{2}$ Jay Redan, MD ${ }^{3}$

'Lotus Clinical Research, Pasadena, CA, USA; ${ }^{2 H}$ eron Therapeutics, San Diego, CA, USA; ${ }^{3}$ AdventHealth Celebration, Celebration, FL, USA

\begin{abstract}
INTRODUCTION
After surgery, up to $70 \%$ of patients experience moderate to severe postoperative pain within the first 72 hours'

Current analgesic treatments include local anesthetics, which provide pain relief for 6 to 12 hours, ${ }^{3}$ and opioid medicines, which are indicated for acute severe pain but can result in serious, even life-threatening adverse
events $(A E s)^{4,5}$

Evidence-based guidelines recommend the use of multimodal analgesia (MMA) to manage postoperative pain

- HTX-0II is an investigational extended-release, dual-acting local anesthetic (DALA) that combines bupivacaine and low-dose meloxicam in a proprietary Biochronomer (Heron Therapeutics, San Diego, CA, USA) extended-release polymer

HTX-OII is administered via needle-free instillation (Figure I) to the surgical site
\end{abstract}

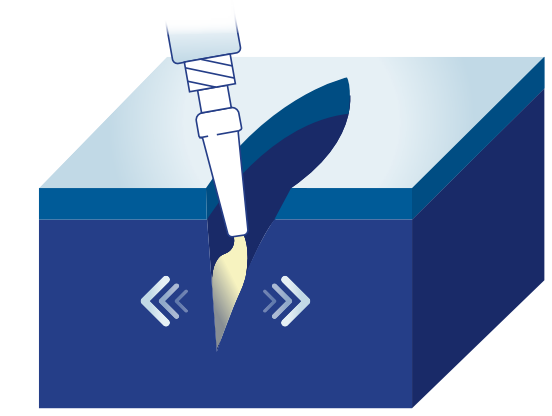

HTX-01I is instilled into the surgitats
needle) and a Luer lock applicato

- In a phase 3 herniorrhaphy study, HTX-011 $300 \mathrm{mg} / 9 \mathrm{mg}$ (bupivacaine/

meloxicam) alone (without a scheduled MMA regimen) (Figure 2)

Relieved severe placebo

Resulted in significanty fewer patients requesting opioid medication

Demonstrated a clinically similar safety profile to bupivacaine $\mathrm{HCl}$
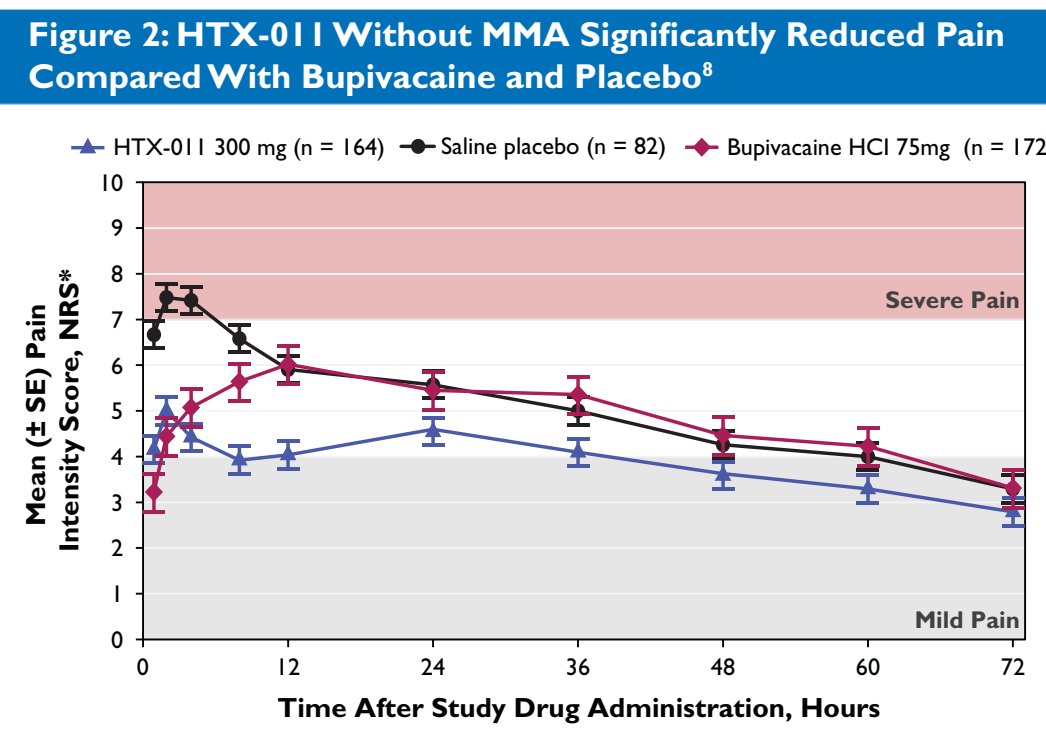

*Using numeric rating scale with windowed worst observation carried forward.
MMA, multimodal analgesia; NRS, numeric rating scale; SE, standard error.

\section{OBJECTIVES}

The study described herein is a follow-on study to the phase 3 herniorrhaphy study and was designed to:

- Evaluate the efficacy of HTX-0II with a non-opioid MMA regimen for postoperative pain, including severe pain, in patients receiving open inguinal herniorrhaphy with mesh

Determine the proportion of opioid-free patients (and total opioid use) after postoperative treatment with HTX-0II as the foundation of a non-opioid MMA regimen

Assess the relationship between severe pain and the use of opioid

medication

Evaluate the safety and tolerability of HTX-0I I with a non-opioid MMA regimen for postoperative pain

\section{METHODS}

- In this open-label study, patients scheduled to undergo open inguinal herniorrhaphy with mesh who met the study entry criteria (Table I) were enrolled

Cohort 2 also received a single dose of intravenous (IV) ketorolac intraoperatively ( $15 \mathrm{mg}$ or $30 \mathrm{mg}$, per product labeling)

Non-opioid preoperative MMA therapy comprised oral acetaminophen $1000 \mathrm{mg}$; non-opioid postoperative MMA therapy comprised oral ibuprofen $600 \mathrm{mg}$ and oral acetaminophen $1000 \mathrm{mg}$ every 6 hours, alternating the 2 medications so that an analgesic was administered
every 3 hours throughout the 72 -hour inpatient postoperative period Patients were kept in the hospital for 72 hours for administration of the scheduled MMA regimen and for assessments of postoperative pain. Opioid rescue medication was available upon request for inadequate pain control independent of pain score

\section{REFERENCES}

Lynch EP et al. Anesth Analg. 1997;85: I 17-123.

2. Svensson I et al.J Pain Symptom Manage. 2000;20:193-20

3. Kehlet $\mathrm{H}$ et al. Acta Anaesthesiol Scand. 201 I;55:778-784.

4. Kessler ER et al. Pharmacotherapy. 2013;33:383-391.

5. Ramachandran SK et al.J Clin Anesthesia. 201 1;23:207-213.

6. American Society of Anesthesiologists. Anesthesiology. 2012;1 16:248-273.

7. Chou R et al.J Pain. 2016;17:131-157.

8. Viscusi E et al. Hernia. 2019. https://doi.org//0.1007/s 10029-019-02023-6.

\begin{tabular}{|c|c|}
\hline Key inclusion criteria & Key exclusion criteria \\
\hline $\begin{array}{l}\text { - Males and females who are not } \\
\text { pregnant or lactating } \\
\text { - Age } \geq 18 \text { years } \\
\text { - Provided written informed } \\
\text { consent } \\
\text { - Scheduled to undergo unilateral } \\
\text { open inguinal herniorrhaphy with } \\
\text { mesh under general anesthesia } \\
\text { - ASA Physical Status Classification } \\
\text { System category I-III }\end{array}$ & $\begin{array}{l}\text { - Pre-existing concurrent acute or } \\
\text { chronic painful/restrictive condition } \\
\text { (unrelated to the hernia) that } \\
\text { may require analgesia during the } \\
\text { postoperative period } \\
\text { - Use of the following within a defined } \\
\text { time period prior to surgery: } \\
\text { NSAIDs ( } 10 \text { days), long-acting opioids } \\
\text { (3 days), any opioid (24 hours), } \\
\text { bupivacaine ( } 5 \text { days), or any local } \\
\text { anesthetic (72 hours) } \\
\text { - } \text { BMI > } 39 \mathrm{~kg} / \mathrm{m}^{2}\end{array}$ \\
\hline
\end{tabular}

ASA,American Society of Anesthesiologists; BMI, body mass index; NSAIDs, nonsteroidal
anti-inflammatory drugs.

Outcome Measures

The primary endpoint was the proportion of patients who did not receive opioid rescue medication (ie, remained opioid-free) through 72 hours after surgery

Secondary endpoints included:

Proportion of patients in severe pain (numeric rating scale $[N R S] \geq 7$ ) at any time through 72 hours after surgery

Total opioid consumption (IV morphine milligram equivalents [MME]) through 72 hours after surgery

Proportion of patients receiving no opioid rescue from 0 through 72 hours who did not require opioid pain medication through day 10 and day 28 recovery

Safety endpoints included incidence of AEs, serious AEs, opioid-related Assessments

Opioid rescue medication taken from time 0 (start of HTX-0II instillation) to 72 hours postsurgery were recorded; patients completed a daily diary to record opioid use (yes/no) from 72 hours through day 28 - Pain level was evaluated at various timepoints using an II-point NRS, where 0 represents "no pain" and 10 represents "worst pain imaginable" Safety was primarily assessed by recording AEs and safety laboratory tests

Statistical Analysis

Opioid-free through 72 hours was defined as 0 MME during the 72-hour postoperative period

Opioid-free from 72 hours through day 10 or 28 was defined as answering "no" to the question "Did you take any opioid medication?" on a daily basis from 72 hours through day 10 or 28 ; patients who reported "yes" or had a missing re

period of interest rescue medication were replaced by the highest pain score recorded befor the opioid was given

\section{RESULTS}

Baseline Population Characteristics

Sixty-three patients (cohort I: 33 patients; cohort 2: 30 patients) were treated with HTX-0II across multiple study sites; 61 (97\%) completed the 72-hour inpatient postoperative period and $58(92 \%)$ completed the study through day 28

Baseline characteristics were well-balanced between cohorts; most

patients were male $(94 \%)$ and the mean BMI was $28 \mathrm{~kg} / \mathrm{m}^{2}$

Pain Intensity

The addition of an intraoperative dose of IV ketorolac did not demonstrate additional benefit; therefore, results for the total study population are presented

Throughout the 72-hour inpatient period, the mean pain intensity score never rose above the mild pain range (NRS $<4$ ) for either cohort (Figure 3 ) The proportion of patients with severe pain (NRS $\geq 7$ ) at any time was low (17.5\%) across both cohorts during the 72 -hour postoperative period; most severe pain occurred during the first 24 hours

The proportion of patients with severe pain was lower in this follow-on study, in which HTX-0II was used as the foundation of a scheduled nonopioid MMA regimen compared with the precedent phase 3 study ${ }^{8}$

\section{Figure 3. HTX-0I I With MMA Kept Mean Pain Intensity in the} Mild Range Through 72 Hours

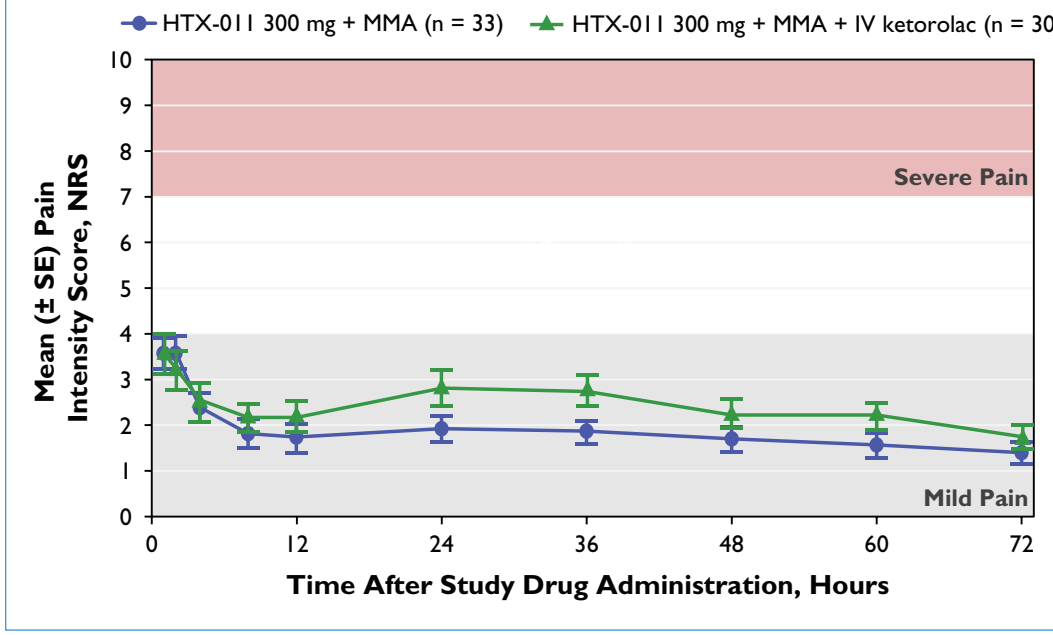

Funding for this research was provided by Heron Therapeutics, Inc. (San Diego, CA, USA). Medical writing assistance was provided by ApotheCom (San Francisco, CA, USA).
Postoperative Opioid Use

$90 \%$ of patients (57/63) did not require opioids to manage pain at any time during the 72-hour inpatient period

Mean total opioid consumption during the 72-hour postoperative period was $<1$ MME in the overall study population and $<10$ MME in the subset of patients who took an opioid during that period

All 6 patients who required opioid rescue medication could be identified early; they reported an NRS score of $\geq 6$ and/or received an opioid within the first 2 hours

The small subset of patients requiring opioid rescue immediately exhibite a distinct pain intensity profile and could be easily identified within the first 2 hours (Figure 4)

\section{Figure 4. Patients Requiring Opioids Have A Distinct Mean Pain}

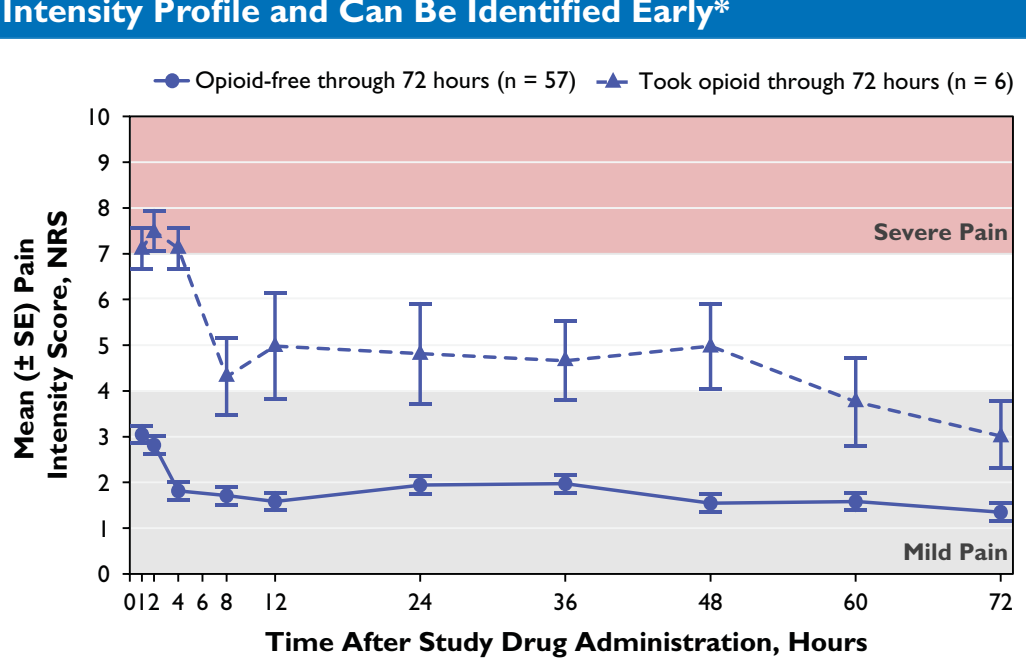

*Combined data from both cohorts.

Safety

Overall, 24 (38\%) patients experienced an AE; the rates of AEs in the two cohorts were similar (Table 2)

The scheduled use of nonsteroidal anti-inflammatory drugs (NSAIDs) did not demonstrate an increase in NSAID-related toxicity

The incidence of TEAEs was much lower in the current study than in the prior phase 3 study, likely due to the lower rates of opioid-related AEs observed in this follow-on study

\begin{tabular}{|c|c|c|c|}
\hline Category & $\begin{array}{c}\text { Cohort I } \\
\text { HTX-0II } \\
n=33\end{array}$ & $\begin{array}{c}\text { Cohort } 2 \\
\text { HTX-0I I + } \\
\text { IV ketorolac } \\
n=30\end{array}$ & $\begin{array}{c}\text { Total } \\
\mathrm{n}=63\end{array}$ \\
\hline Any AE & $12(36.4)$ & $12(40.0)$ & $24(38.1)$ \\
\hline $\begin{array}{l}\text { AE possibly related to } \\
\text { HTX-01I }\end{array}$ & $2(6.1)$ & I (3.3) & $3(4.8)$ \\
\hline Opioid-related AE & $2(6.1)$ & $5(16.7)$ & $7(11.1)$ \\
\hline $\begin{array}{l}\text { AE leading to } \\
\text { premature withdrawal }\end{array}$ & 0 & 0 & 0 \\
\hline Fatal or other SAE & 0 & 0 & 0 \\
\hline
\end{tabular}

\section{SUMMARY AND CONCLUSIONS}

HTX-0Il, in combination with a scheduled regimen of non-opioid overthe-counter analgesics, eliminated severe postoperative pain in $82.5 \%$ of patients

The mean pain intensity score did not rise above the mild range at any time during the 72-hour inpatient period

Patients who experienced severe pain did so within the first 2 hours after surgery

Pain intensity and opioid use were similar between cohorts, and no additional benefit of IV ketorolac was observed

The majority (90\%) of patients did not require opioids to manage pain at any time during the 72-hour inpatient period; of these patients, most (91\%) remained opioid-free through recovery at day 28

Based on these results, the following algorithm was developed to determine who should receive a discharge prescription for opioids in future studies

Patients who receive an opioid rescue or have a NRS pain score of

$\geq 6$ within the first 2 hours after surgery

The combination of HTX-0II and the scheduled non-opioid MMA regimen was well tolerated, and use of NSAIDs did not increase NSAID-related toxicity

These data demonstrate that HTX-OII could be the foundation for an opioid-free post-operative recovery 